\title{
Optimization of Landscape Phage Fusion Protein-Modified Polymeric PEG-PE Micelles for Improved Breast Cancer Cell Targeting
}

Tao Wang ${ }^{1}$, Valery A. Petrenko ${ }^{2}$ and Vladimir P. Torchilin ${ }^{1 *}$

${ }^{1}$ Center for Pharmaceutical Biotechnology and Nanomedicine, Northeastern University, Boston, MA 02115, USA

${ }^{2}$ Department of Pathobiology, College of Veterinary Medicine, Auburn University, AL 36849, USA

\begin{abstract}
Amphiphilic landscape phage fusion proteins with high affinity and selectivity towards breast cancer MCF-7 (Michigan Cancer Foundation-7) cells self-assemble with polymeric PEG-PE conjugates to form mixed micelles (phagemicelles) capable of cancer cell-targeted delivery of poorly-soluble drugs. While the PEG corona provides the stability and longevity to the micelles, its presence is a potential steric difficulties for the interaction of phage fusion protein with cell surface targets. We attempted to address this problem by controlling the length of the PEG block and the phage fusion protein quantity, selecting the optimal ones to produce a reasonable retention of the targeting affinity and selectivity of the MCF-7-specific phage fusion protein. Three PEG-PE conjugates with different PEG lengths were used to construct phage- and plain-micelles, followed by FACS analysis of the effect of the PEG length on their binding affinity and selectivity towards target MCF-7 cells using either a MCF-7 cell monoculture or a cell co-culture model composed of target cancer MCF-7 cells and non-target, non-cancer C166 cells expressing GFP (Green Fluorescent Protein). Both, the length of PEG and quantity of phage fusion protein had a profound impact on the targetability of the phage-micelles. Phage-micelles prepared with $\mathrm{PEG}_{2 \mathrm{k}}-\mathrm{PE}$ achieved a desirable binding affinity and selectivity. Incorporation of a minimal concentration of phage protein, up to $0.5 \%$, produced maximal targeting efficiency towards MCF-7 cells. Overall, phage-micelles with $\mathrm{PEG}_{2 \mathrm{k}}-\mathrm{PE}$ and $0.5 \%$ of phage protein represent the optimal formulation for targeting towards breast cancer cells.
\end{abstract}

Keywords: Drug delivery; Polymeric micelles, PEG-PE; Phage display; Landscape phage fusion protein; Tumor targeting; Breast cancer

\section{Introduction}

Lipophilic compounds account for more than $40 \%$ of new drug candidate molecules. Despite their potent pharmacological activity, therapeutic application of these molecules is limited due to their poor solubility and low bioavailability. To overcome this limitation, many efforts have been made to develop effective drug delivery systems in order to enhance solubility of hydrophobic drugs [1-4].

One of promising delivery systems is the polymeric micelle. Nano-scale micellar particles can be formed by the self-assembly of amphiphilic molecules in an aqueous environment, with hydrophobic fragments forming the core of a micelle and with the hydrophilic parts forming a micellar corona $[5,6]$. The hydrophobic core of a micelle has been used to encapsulate a variety of sparingly-soluble therapeutic and diagnostic agents [5-9]. Micellar drug delivery systems substantially increase the bioavailability of poorly-soluble pharmaceuticals and protect them from destructive factors upon parenteral administration [10]. Their nanometre-sizes (typically, between 5 and $50 \mathrm{~nm}$ ) allow micellar drugs to passively target tumor sites via the Enhanced Permeability and Retention (EPR) effect [11].

The tumor-targeting efficiency of micelle-encapsulated drugs can be further enhanced by introducing targeting ligands into a micellar formulation to allow for active targeting of tumors $[6,12]$. In the ongoing efforts in the search for targeting ligands, peptide-mediated tumor targeting has become a fast growing field [13], since peptides show multiple advantages, including lesser susceptibility to clearance by the Mononuclear Phagocyte System (MPS), less immunogenicity, and better tumor penetration when compared with antibodies $[14,15]$. The molecular mechanism behind this phenomenon is that peptide receptors are over-expression in a wide spectrum of tumors [16].
Combinatorial technologies, such as phage display techniques, have made targeting ligands available in a high throughput fashion $[17,18]$.

Recently, we identified MCF-7 breast- and prostate cancer-specific phage fusion proteins and demonstrated their abilities for activelytargeted delivery of the liposomal doxorubicin [19-22]. However, the effectiveness of the use of liposomes for delivery of water-insoluble drugs is far from ideal. Both, the limited drug encapsulation capacity and the potentially unstable packaging of hydrophobic drugs within the lipid bilayers of liposomes are major concerns [5,23]. Additionally, the in vivo premature release of a liposome-loaded hydrophobic drug could represent other issues [23]. We proposed recently the use of PEG-PE-based micelles for the solubilization of hydrophobic drugs [12] and explored the utility of phage fusion proteins in targeting delivery of micelles carrying poorly-soluble drugs. This approach is based on the amphiphilic nature of the phage fusion protein, which ensures its ability to assemble spontaneously with micelle-forming polymers, such as PEG-PE conjugates, resulting in the formation of mixed micelles capable of delivery of water-insoluble drug to specific tumor cells (Figure 1).

However, the presence of a PEG corona in the mixed micelles could potentially set up a steric barrier between the targeting phage fusion

*Corresponding author: Vladimir P. Torchilin, Center for Pharmaceutical Biotechnology and Nanomedicine, Northeastern University, Boston, MA 02115 USA, Tel: 617373 3206; Fax: 617373 7509; E-mail:v.torchilin@neu.edu

Received March 06, 2012; Accepted April 17, 2012; Published April 20, 2012

Citation: Wang T, Petrenko VA, Torchilin VP (2012) Optimization of Landscape Phage Fusion Protein-Modified Polymeric PEG-PE Micelles for Improved Breast Cancer Cell Targeting. J Nanomedic Nanotechnol S4:008. doi:10.4172/2157-7439. S4-008

Copyright: ( 2012 Wang T, et al. This is an open-access article distributed under the terms of the Creative Commons Attribution License, which permits unrestricted use, distribution, and reproduction in any medium, provided the original author and source are credited. 


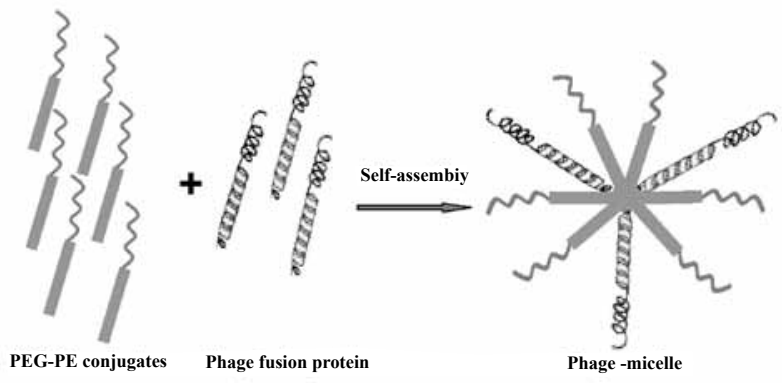

Figure 1: Schematic representation of the self-assembly of landscape phage fusion protein with polymeric PEG-PE conjugates to form phage-micelles for targeted delivery of hydrophobic drugs.

peptides and molecular receptors on the surface of target cells. In this study, we sought to address this problem by controlling the length of the PEG block and phage protein quantity, selecting parameters, which produce an optimal balance between the target affinity and selectivity of the micelle-incorporated phage protein.

\section{Materials and Methods}

\section{Materials and reagents}

1,2-distearoyl-sn-glycero-3-phosphoethanolamine-N[amino(polyethylene glycol)2000] (ammonium salt; $\mathrm{PEG}_{2000}$-PE), 1,2-distearoyl-sn-glycero-3-phosphoethanolamine- $\mathrm{N}$-[amino(polyethylene glycol)750] (ammonium salt; $\mathrm{PEG}_{750}$-PE), 1,2-distearoyl-sn-glycero-3phosphoethanolamine- $\mathrm{N}$-[amino(polyethylene glycol)5000] (ammonium salt; $\mathrm{PEG}_{5000} \mathrm{PE}$ ), and 1,2-dimyristoyl-sn-glycero-3-phosphoethanolamine- $\mathrm{N}$-(lissamine rhodamine $\mathrm{B}$ sulfonyl) (ammonium salt, Rho-PE) were purchased from Avanti Polar Lipids Inc (Alabaster, AL). Paclitaxel (PCT) was from Cedarburg Pharmaceuticals (Beverly, MA). Dimethyl sulfoxide (DMSO) was purchased from Fisher Scientific (Pittsburgh, PA). Sodium cholate was from Sigma (St.Louis, MO). Cell Titer Blue assay kit was from Promega (Madison, WI). Fluor Mounting Medium was from Trevigen Inc (Gaithersburg, MD). MCF-7 human breast adenocarcinoma (HTB-22 ${ }^{\mathrm{TM}}$ ) cells and C166-GFP (CRL-2583 ${ }^{\mathrm{TM}}$ ) mouse yolk sac endothelial cells were obtained from the ATCC (American Type Culture Collection) (Manassas, VA). All cells were grown as recommended by the ATCC at $37^{\circ} \mathrm{C}, 5 \% \mathrm{CO}_{2}$.

\section{Phage fusion protein}

Phage selection and phage protein purification have been carried out as described by us previously [19].

\section{Preparation of micellar formulations}

Phage-micelles were prepared with compositions shown in Table 1. Briefly, to make rhodamine-labeled micelles, $5 \mathrm{mM}$ of PEG-PE was mixed with traces of rhodamine-PE in chloroform. To form phagemicelles, after evaporation of chloroform, the PEG-PE film formed was hydrated with MCF-7-specific-phage fusion proteins dissolved in $10 \mathrm{mM}$ of sodium cholate, followed by vortexing and overnight dialysis against Phosphate Buffered Saline (PBS), $\mathrm{pH} 7.4$ to remove the detergent. To form plain micelles, the PEG-PE film formed after evaporation was hydrated with $\mathrm{PBS}, \mathrm{pH} 7.4$, followed by vortexing and overnight dialysis against PBS, $\mathrm{pH}$ 7.4.

\section{FACS analysis of the uptake of phage-micelles by MCF-7 Cells}

MCF-7 cells were grown in $12.5 \mathrm{~cm}^{2}$ flasks in MEM with $10 \%$ serum until $70-80 \%$ confluence. Cells were incubated with $75 \mu \mathrm{M}$ of rhodamine-labeled plain- or phage-micelle formulations for $1 \mathrm{~h}$. After washing 3 times with PBS, pH 7.4, cells were detached and collected by centrifugation. The cell pellets were resuspended in $200 \mu$ of PBS with $4 \%$ paraformaldehyde, followed by flow cytometry (FACS) analysis. A right shift on the $\mathrm{x}$-axis of the histogram plot indicated the cellular binding of the rhodamine-labeled micelles.

\section{FACS Analysis of Selective Binding of Phage-Micelles with Target MCF-7 Cells in a Co-culture Model}

Target MCF-7 cells were co-cultured with non-target C166 endothelial cells expressing GFP in a $1: 1$ ratio and seeded in $12.5 \mathrm{~cm}^{2}$ flasks in MEM with $10 \%$ serum. After co-culture until $70-80 \%$ confluence, cells were incubated with $75 \mu \mathrm{M}$ of rhodamine-labeled plain- or phage-micelle formulations for $1 \mathrm{~h}$. The cells were washed 3 times with PBS ( $\mathrm{pH}$ 7.4), detached, and collected by centrifugation. The cell pellets were resuspended in $200 \mu \mathrm{l}$ of PBS with $4 \%$ paraformaldehyde, followed by the FACS analysis. After acquiring data displayed as dot plots, the dot plots were inserted into four regions (R1, R2, R3, and R4). The cellular binding of the rhodamine-labeled micelles was detected as a right shift of the cell population on the $\mathrm{x}$ axis (FL2-H, Red). The percent cell-associated micelles were calculated as follows:

For MCF-7 cells,

The percent cells with associated micelles $=\mathrm{R} 3 /(\mathrm{R} 1+\mathrm{R} 3) \times 100$.

\section{For C166-GFP cells,}

The percent of cells with associated micelles $=\mathrm{R} 4 /(\mathrm{R} 2+\mathrm{R} 4) \times 100$.

The binding affinity was determined by the percent of MCF-7 cells with associated micelles.

The binding selectivity was defined as the percent of MCF-7 cells with associated micelles divided by the percent of C166-GFP cells with associated micelles as follows:

The binding selectivity $=[\mathrm{R} 3 /(\mathrm{R} 1+\mathrm{R} 3)] /[\mathrm{R} 4 /(\mathrm{R} 2+\mathrm{R} 4)]$.

\section{Fluorescence microscopy analysis of selective binding of optimized phage-micelles to target MCF-7 cells}

MCF-7 and C166-GFP cells were co-cultured on 6-well plates for $24 \mathrm{~h}$ at $37^{\circ} \mathrm{C}$, and treated with $75 \mu \mathrm{M}$ of rhodamine-labeled phagemicelles in MEM with $10 \%$ serum for $30 \mathrm{~min}$ at $37^{\circ} \mathrm{C}$. After washing 3 times with PBS, the coverslip was mounted onto a glass slide over the fluorescence mounting medium. The images were acquired by a fluorescence microscope (Nikon, Japan) at $40 \times$ magnification with FITC or TRITC filters.

\section{Cytotoxicity}

After MCF-7 cells were cultured in 96-well microplates to 50-60\% confluence, cells were treated for $72 \mathrm{~h}$ with paclitaxel (PCT)-loaded, optimized $\mathrm{PEG}_{2 \mathrm{k}}-\mathrm{PE}$ phage-micelles and different controls, including free PCT in DMSO, PCT-loaded PEG $_{2 \mathrm{k}}$-PE plain micelles, and drugfree, optimized phage-micelles. The concentration of paclitaxel used in different treatments with drug-containing formulations is equivalent to $1.76 \mu \mathrm{M}$. Cells were then washed once with PBS, $\mathrm{pH} 7.4$, and incubated with fresh complete medium $(100 \mu \mathrm{l} /$ well $)$ along with the Cell Titer Blue assay reagent $(20 \mu \mathrm{l} /$ well $)$ for $2 \mathrm{~h}$ at $37^{\circ} \mathrm{C}$. The fluorescence intensity was measured using a multi-detection microplate reader (Bio-Tek, Winooski, VT) with 525/590 nm excitation/emission wavelengths. 
Citation: Wang T, Petrenko VA, Torchilin VP (2012) Optimization of Landscape Phage Fusion Protein-Modified Polymeric PEG-PE Micelles for Improved Breast Cancer Cell Targeting. J Nanomedic Nanotechnol S4:008. doi:10.4172/2157-7439.S4-008

Page 3 of 6

\begin{tabular}{|c|c|c|c|c|}
\hline The use of micelles & Designations & PEG length & $\begin{array}{l}\text { PEG-PE } \\
(\mathrm{mM})\end{array}$ & $\begin{array}{l}\text { Phage Protein } \\
(\% \mathrm{w} / \mathrm{w})\end{array}$ \\
\hline \multirow[t]{6}{*}{${ }^{*}$ Optimization of PEG length } & Phage PEG5K-PE micelles & $5 \mathrm{~K}$ & 5 & 0.5 \\
\hline & Phage PEG2K-PE micelles & $2 \mathrm{~K}$ & 5 & 0.5 \\
\hline & Phage PEG750-PE micelles & 750 & 5 & 0.5 \\
\hline & Plain PEG5K-PE micelles & $5 \mathrm{~K}$ & 5 & 0 \\
\hline & Plain PEG2K-PE micelles & $2 \mathrm{~K}$ & 5 & 0 \\
\hline & Plain PEG750-PE micelles & 750 & 5 & 0 \\
\hline \multirow[t]{5}{*}{ *Optimization of phage protein quantity } & Plain PEG2K-PE micelles & $2 \mathrm{~K}$ & 5 & 0 \\
\hline & Phage PEG2K-PE micelles & $2 \mathrm{~K}$ & 5 & 0.125 \\
\hline & Phage PEG2K-PE micelles & $2 \mathrm{~K}$ & 5 & 0.25 \\
\hline & Phage PEG2K-PE micelles & $2 \mathrm{~K}$ & 5 & 0.5 \\
\hline & Phage PEG2K-PE micelles & $2 \mathrm{~K}$ & 5 & 1 \\
\hline
\end{tabular}

${ }^{*}$ Trace rhodamine-PE was added to formulations
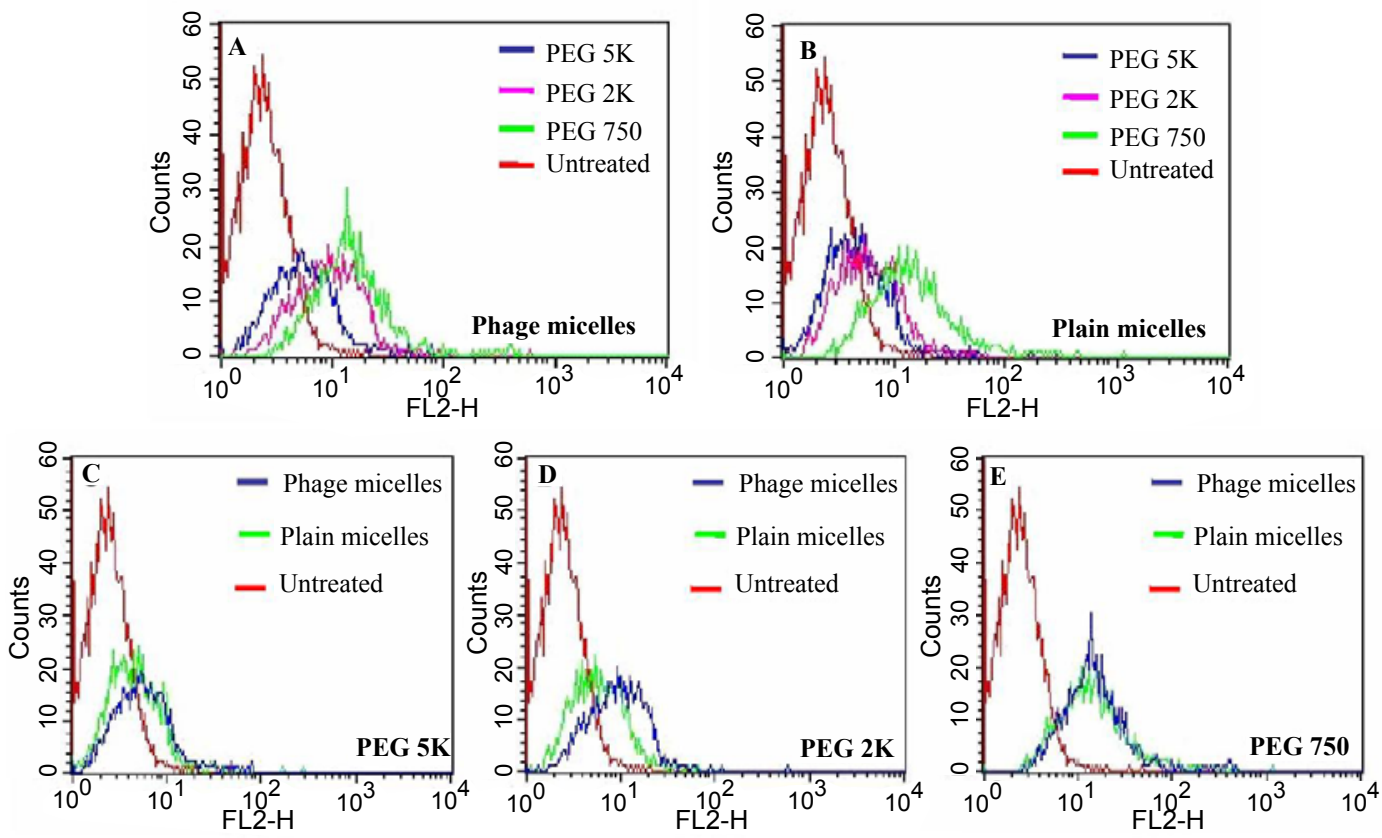

Figure 2: FACS analysis of the effect of different PEG length on the binding affinity of micellar formulations towards target MCF-7 cells. MCF-7 breast cancer cells were treated for $1 \mathrm{~h}$ with either plain micelles or phage-micelles constructed from $\mathrm{PEG}_{750}-\mathrm{PE}, \mathrm{PEG}_{2 \mathrm{k}}-\mathrm{PE}$, or $P E G_{5 \mathrm{k}}-\mathrm{PE}$ conjugates, followed by $\mathrm{FACS}$ analysis of cell-associated micelles as indicated by an increase in red fluorescence intensity (FL2-H).

\section{Results}

\section{Optimization of the PEG Length}

We have used three PEG species with different lengths to construct plain- and phage-micelles, and compared their binding affinity towards target MCF-7 cells. As expected, a decrease in the length of PEG chain increased target cell association of phage-micelles in the order of $\mathrm{PEG}_{750}$ $>\mathrm{PEG}_{2 \mathrm{k}}>\mathrm{PEG}_{5 \mathrm{k}}$ (Figure 2A). Control plain micelles with varying PEG chain also showed their binding affinity to MCF-7 cells in the order of $\mathrm{PEG}_{750}>\mathrm{PEG}_{2 \mathrm{k}}>=\mathrm{PEG}_{5 \mathrm{k}}$ (Figure $2 \mathrm{~B}$ ). However, the modification of $\mathrm{PEG}_{5 \mathrm{k}}$ micelles (Figure 2C) and $\mathrm{PEG}_{750}$ (Figure 2E) micelles with MCF-7 specific phage fusion protein showed less advantage in tumorcell targeting compared to non-modified plain micelles. In contrast, the phage protein-modified $\mathrm{PEG}_{2 \mathrm{k}}$-PE micelles (Figure 2D) showed a significantly enhanced target cell binding compared to plain $\mathrm{PEG}_{2 \mathrm{k}}-\mathrm{PE}$ micelles.
To investigate the binding selectivity of different micelle formulations towards target MCF-7 cancer cells compared to nontarget, non-cancer cells, we have designed a co-culture assay, in which target cancer MCF-7 cells were co-grown with non-target, non-cancer endothelial cells, C166 cells expressing GFP (C166-GFP). FACS analysis of the co-culture revealed two distinct cell populations. One cell population corresponding to higher green fluorescence intensity, as indicated on the Y-axis (FL1-H) of the dot plot, was C166-GFP cells. The other cellular population with lower green fluorescence intensity was MCF-7 cells (Figure 3A). After Treatment of this co-culture with different micelles followed by the FACS analysis, we found that plainand phage- $\mathrm{PEG}_{750}$-PE micelles had the highest binding to target cells but also to non-target cells, indicating a poor selective binding to tumor cells (Figure 3B and Figure 3E), while micelles with $\mathrm{PEG}_{5 \mathrm{~K}}-\mathrm{PE}$ showed the best targeting selectivity but a compromised binding affinity (Figure $3 \mathrm{D}$ and Figure $3 \mathrm{G})$. Micelles with $\mathrm{PEG}_{2 \mathrm{k}}-\mathrm{PE}$ had an optimal balance 
Citation: Wang T, Petrenko VA, Torchilin VP (2012) Optimization of Landscape Phage Fusion Protein-Modified Polymeric PEG-PE Micelles for Improved Breast Cancer Cell Targeting. J Nanomedic Nanotechnol S4:008. doi:10.4172/2157-7439.S4-008

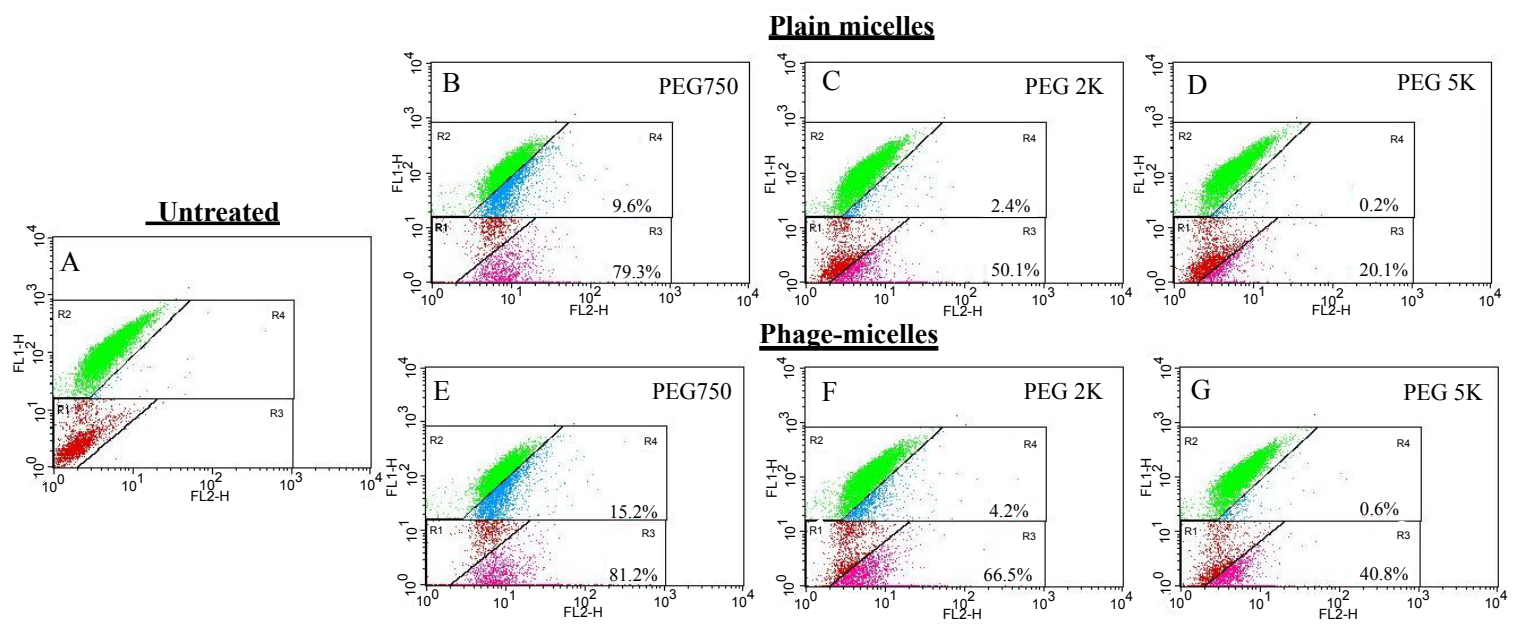

Figure 3: FACS analysis of cellular binding of micellar formulations prepared with different PEG blocks in a co-culture system. A co-culture composed of target MCF-7 cells and non-target C166-GFP cells was treated for $1 \mathrm{~h}$ with either plain micelles or phage-micelles constructed from $P E G_{750}-P E, P G_{2 K}-P E$, or $P E G_{5 K}-P E$ conjugates, followed by FACS analysis of cell-associated micelles as indicated by the red fluorescence increase. The dot plots were bounded into four regions (R1, R2, R3, and R4). FL1-H (green); FL2-H (red); (A) A representative dot plot showing the untreated co-culture of MCF-7 cells and C166-GFP cells. Red dots in region $\mathrm{R} 1$, the location of untreated MCF-7 cells; green dots in region R2, the location of untreated C166-GFP cells; (B-D) Plain micelle-treated co-culture of MCF-7 cells and C166-GFP cells. Pink dots in region R3, plain-micelle-associated MCF-7 cells; blue dots in region R4, plain-micelle-associated C166-GFP cells; (E-G) MCF-7-targeted phage-micelle-treated co-culture of MCF-7 and C166-GFP cells. Pink dots in region R3, phage-micelle-associated MCF-7 cells; blue dots in R4, phage-micelleassociated C166-GFP cells.

A

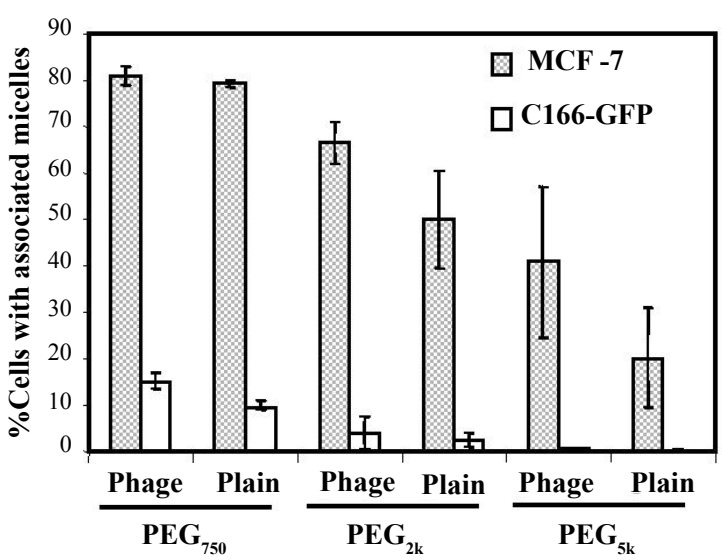

B

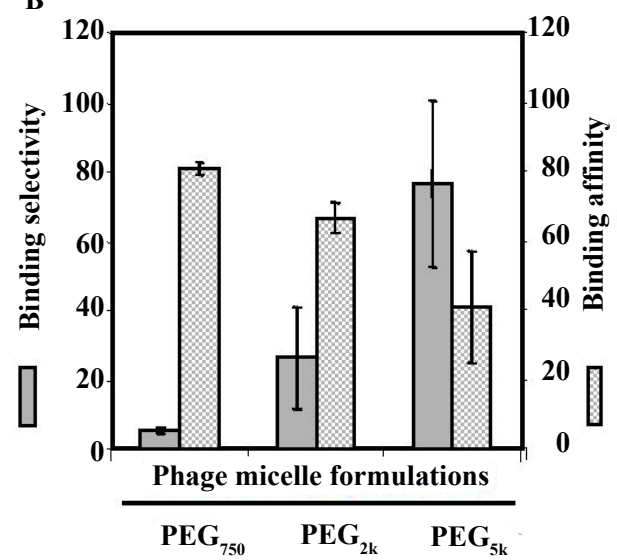

Figure 4: Quantitative analysis of the effect of PEG length on the binding affinity and selectivity of micellar formulations after FACS analysis in a cocultured cell model. (A) The percent of cells with associated micelles indicated as the cell population shifted to the region with higher red fluorescence intensity in the dot plot acquired by FACS analysis. (B) The binding affinity defined as the percent of MCF-7 cells with associated micelles divided by the total MCF-7 cells tested; The binding selectivity defined as the percent of MCF-7 cells with associated micelles divided by the percent of C166-GFP cells with associated micelles (mean $\pm \mathrm{SD}, \mathrm{n}=9$ )

between the binding affinity and selectivity (Figure 3C, Figure $3 \mathrm{~F}$ and Figure 4).

\section{Optimization of phage protein quantity}

We defined an optimal phage protein quantity as one providing the maximal binding affinity with a minimum of phage fusion protein used. The incorporation of MCF-7-targeting phage fusion protein up to $0.25 \%$ by weight into $P_{2 G_{2 k}}$-PE micelles resulted in a limited increase in target cell-association compared to the plain $\mathrm{PEG}_{2 \mathrm{k}}$-PE micelles, but the modification of plain micelles with $0.5 \%$ phage fusion protein provided a pronounce enhancement in target MCF-7 cell-binding, but the further increase in phage protein quantity from $0.5 \%$ to $1 \%$ by weight did not result in any noticeable increase in the binding (Figure 5). Therefore, the optimal phage protein quantity to be used in phagemicelles is $0.5 \%$ by weight.

\section{Targetability and cytotoxicity of the optimized phage-micelle formulation}

After treatment of the co-culture composed of target MCF-7 and non-target C166-GFP with rhodamine-labeled phage-micelles, the overlay fluorescence micrograph clearly showed the lack of colocalization between red and green fluorescence, indicating that the 


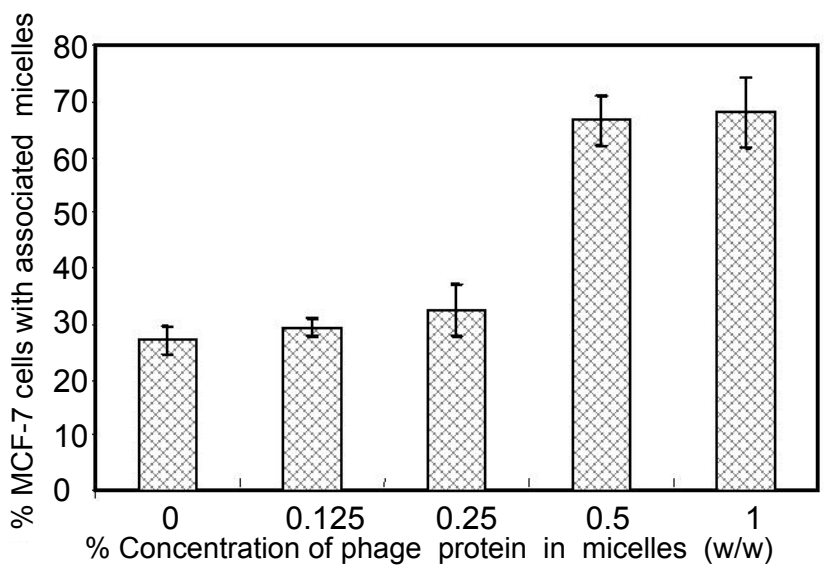

Figure 5: Effect of phage protein quantity on the binding affinity of micelle formulations towards target MCF-7 cells (Mean $\pm S D, n=6$ ).

optimized phage-micelles preferentially bind to target MCF-7 cancer cells rather than to co-cultured C166-GFP cells (Figure 6A).

The treatment with the optimized phage-micelles loaded with paclitaxel at a PCT concentration of $1.76 \mu \mathrm{M}$ for $72 \mathrm{~h}$, led to $51.3 \%$ MCF-7 cell death. The estimated $\mathrm{IC}_{50}$ of the phage micelle-loaded with paclitaxel is $1.63 \mu \mathrm{M}$ (as PCT). The significantly lower tumor cell killing is seen however in the groups treated with both, free paclitaxel dissolved in DMSO and paclitaxel in plain $\mathrm{PEG}_{2 \mathrm{k}}-\mathrm{PE}$ micelles at the equivalent concentration of PCT tested, with MCF-7 cell death level at $18.7 \%$ and $23.8 \%$, respectively (Figure $6 \mathrm{~B}$ ). The drug-free, optimized phage-micelles produced negligible tumor cell killing.

\section{Discussion}

Combining of passive tumor targeting of pharmaceutical nanocarriers with tumor cell recognition systems represents a sophisticated strategy for targeted delivery of anti-cancer drugs to specific tumor cells [24]. Within this approach, a novel self-assembled micellar system, composed of PEG-diacyllipid micelles and phagederived fusion proteins, has recently been designed for tumor-targeted delivery of water-insoluble drugs, such as paclitaxel [12].

The PEG-diacyllipid micelles prepared from amphiphilic polymer conjugates of polyethylene glycol (PEG) and diacyllipids (PE) have served as drug carriers for the solubilization of water-insoluble drugs and for tumor passive targeting $[5,6,10]$. Compared with conventional amphiphilic polymer micelles, the use of diacyllipid moieties (PE) as hydrophobic blocks, offers better particle stability as a result of a considerable contribution of the two fatty acid acyls within the PE blocks that increase hydrophobic interactions within the micelle's core. On the other hand, highly water-soluble PEG chains effectively provide steric protection and physiological stability for various nanoparticles in biological media. PEG chains protect nanoparticles from the clearance by the MPS system, and from other possible undesirable interaction with blood components. In the blood stream, PEGylated particles have a longer circulation time, which additionally promotes drug accumulation within target sites, such as tumors, via the EPR effect. Consequently, PEG-PE micelles loaded with a variety of poorly-soluble drugs can deliver their payload into tumors in mice with a greater efficiency $[25,26]$.
It has been recognized for some time that PEGylation could also influence drug delivery negatively $[27,28]$. The presence of the PEG corona covering drug carriers produces a steric hindrance, affecting the interaction of drug carriers with target cells [28]. Particularly, PEG blocks within the micelles modified with phage fusion protein could have interfered with the interaction of targeting phage fusion protein with tumor cell-surface receptors.

An important method to solve this PEG dilemma is to control PEG length to induce a proper balance between the micellar stability and its targetability. Early studies have shown the effect of PEG length on micellar size and thermodynamic stability [5]. Generally, PEG-PE conjugates with PEG blocks with a molecular weight from 1000 to 15000 Dalton are able to form stable nanoparticles. With an increase in the length of PEG form 750 to 2000 to 5000 Dalton, particle sizes are increased and the Critical Micellar Concentration (CMC) of PEGPE micelles is decreased [5]. Further studies have demonstrated that PEG length also dictates the in vivo behaviour of PEG-PE micelles. Micelles formed with $\mathrm{PEG}_{5 \mathrm{~K}}-\mathrm{PE}$ have a longer circulation time, with less uptake by normal tissue compared to micelles prepared from a shorter PEG-PE conjugate, and a higher accumulation in tumors compared to non-target tissues, such as muscle, as observed in a Lewis lung carcinoma-bearing experimental mice $[29,30]$. Our results have further demonstrated the shielding effect produced by the PEG chain.

A

\section{Coculture of MCF-7 \& C166-GFP}
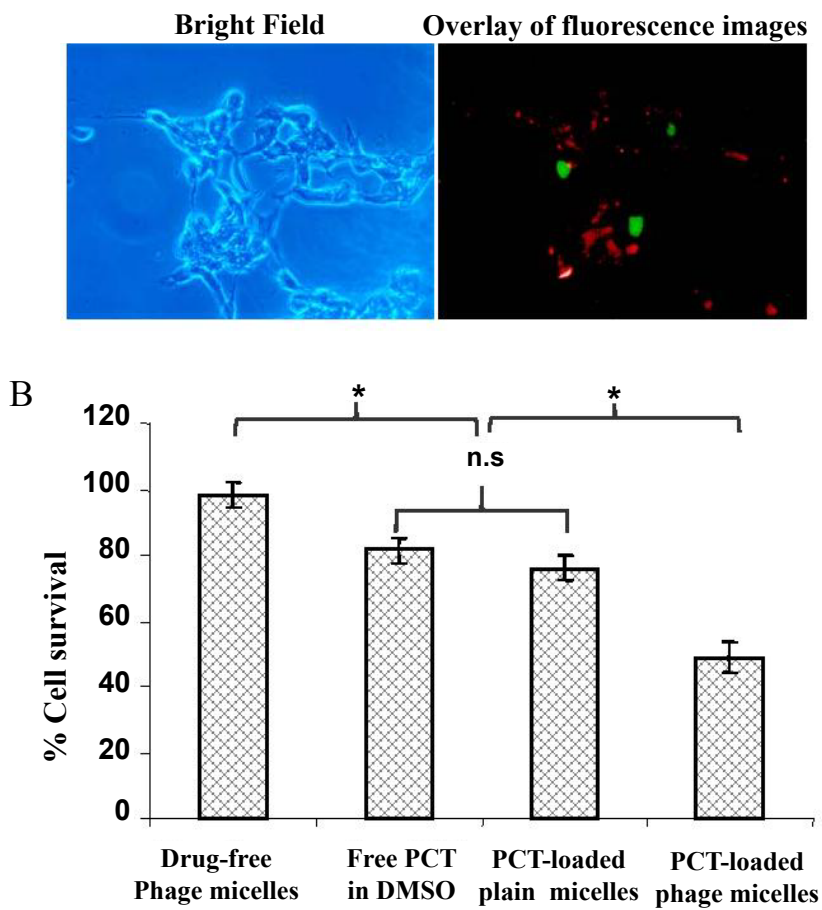

Figure 6: Targetability and cytotoxicity of the optimized phage-micelle formulation. (A) Selective cell binding by the florescence microscopy. Bright field shows a co-culture of MCF-7 and C166-GFP cells. In the overlay image, green fluorescence shows the location of C166-GFP cells; red fluorescence shows rhodamine-labeled phage-micelles associated with cells. (B) Cytotoxicity of different formulations towards MCF-7 cells after $72 \mathrm{~h}$ treatment with MCF7-targeted phage-micelles and various controls, including free PCT in DMSO, PCT-loaded plain micelles, drug-free MCF-7-targeted phage-micelles. ( ${ }^{*} \mathrm{p}$ $<0.05$, mean $\pm S D, n=6$ ) 
Citation: Wang T, Petrenko VA, Torchilin VP (2012) Optimization of Landscape Phage Fusion Protein-Modified Polymeric PEG-PE Micelles for Improved Breast Cancer Cell Targeting. J Nanomedic Nanotechnol S4:008. doi:10.4172/2157-7439.S4-008

Page 6 of 6

The longer the PEG block used, the weaker the interaction of PEG-PE micelles with tumor cells. On the other hand, micelles prepared from PEG-PE conjugates with longer versions of PEG have better targeting selectivity towards tumor cells compared to non-target, non-cancer cells.

Within the objective of this study, to develop an optimized selfassembling system for active tumor targeting, we used the whole landscape phage fusion protein (the targeting peptide fused to phage major coat protein PVIII), the hydrophobic phage major coat protein part of which interacts with diacyllipid-PE to form the micellar core. Targeting peptides of the whole protein, together with PEG block of PEG-PE conjugates, built up the micellar corona. An ideal phagemicelle formulation should contain an appropriate PEG block, which can efficiently shield hydrophobic segments (PE and phage major coat protein PVIII), and, at the same time, exposes the targeting peptides to a maximally possible extent. During optimization of the PEG length and phage protein quantity used to formulate the targeted phage-micelles, we found that only $\mathrm{PEG}_{2 \mathrm{~K}}$-PE increased binding affinity of phagemicelles towards targeted cells, implying that $\mathrm{PEG}_{750}$ was too short to hide the hydrophobic segments, while $\mathrm{PEG}_{5 \mathrm{~K}}$ was too long to allow for the exposure of targeting ligands. In accordance with this observation, $\mathrm{PE} \mathrm{G}_{750}$-PE micelles showed pronounced non-specific binding to nontarget C166-GFP cells. Interestingly, the increase in phage protein quantity in $\mathrm{PEG}_{2 \mathrm{~K}}$-PE phage-micelles was not necessarily favourable for targeting, suggesting that the mixed micellar aggregations can only accommodate a certain amount of phage fusion protein within stable particles with a maximal tumor cell targeting. Similar phenomenon was also observed when the phage protein was used to modify liposomes [31].

Loading hydrophobic drug paclitaxel into the optimized formulation with $\mathrm{PEG}_{2 \mathrm{~K}} \mathrm{-PE}$ and $0.5 \%$ of phage fusion protein (by weight) forms stable nanoparticles. Incorporation of phage protein into $\mathrm{PEG}_{2 \mathrm{~K}}-\mathrm{PE}$ micelles improves MCF-7 tumor cell targeting and cytotoxicity of the micellar paclitaxel [12]. Similarly, we have earlier observed the specific tumor cell targeting and enhanced tumor cell killing when liposomes are modified with phage fusion protein for the delivery of water-soluble amphiphilic drugs, such as doxorubicin hydrochloride [19].

The $\mathrm{PEG}_{2 \mathrm{~K}}$-DSPE used in the preparation of the optimized phagemicelles has been approved as an excipient by the U.S. Food and Drug Administration, and it is generally safe, biocompatible and relatively nontoxic $[32,33]$. Thorough investigation on the metabolism and excretion of PEG and PEG-biological molecule conjugates suggests the less safety concerns associated with their utility in chronic and acute administration even with high concentration [34]. In this study, drugfree PEG-PE micelles modified with phage fusion protein showed no toxicity towards MCF-7 at the concentration of PEG-PE up to $48 \mu \mathrm{M}$, providing evidence for the safe use of the phage-micelle formulation.

In summary, phage-micelles created by the self-assembly of phage fusion protein and PEG-PE specifically bind to targeted tumor cells. Both length of PEG and quantity of phage fusion protein have a profound impact on the micellar targetability. $\mathrm{PEG}_{2 \mathrm{~K}}-\mathrm{PE}$ and phage protein quantity of $0.5 \%(\mathrm{w} / \mathrm{w})$ constitute a formulation with an optimized balance between tumor cell-binding affinity and selectivity.

\section{Acknowledgements}

This work was supported by NIH grant \# R01 CA125063-01 and the Animal Health and Disease Research grant 2006-9, College of Veterinary Medicine, Auburn University to Valery A. Petrenko and by NIH grants U54CA151881 to Vladimir P. Torchilin.

\section{References}

1. Merisko-Liversidge EM, Liversidge GG (2008) Drug nanoparticles: formulating poorly water-soluble compounds. Toxicol Pathol 36: 43-48.

2. Dahan A, Hoffman A (2008) Rationalizing the selection of oral lipid based drug delivery systems by an in vitro dynamic lipolysis model for improved ora bioavailability of poorly water soluble drugs. J Control Release 129: 1-10.

3. Brewster ME, Loftsson $\mathrm{T}$ (2007) Cyclodextrins as pharmaceutical solubilizers Adv Drug Deliv Rev 59: 645-666.

4. Porter CJ, Trevaskis NL, Charman WN (2007) Lipids and lipid-based formulations: optimizing the oral delivery of lipophilic drugs. Nat Rev Drug Discov 6: 231-248.

5. Torchilin VP (2007) Micellar nanocarriers: pharmaceutical perspectives. Pharm Res 24: 1-16.

6. Torchilin VP (2007) Targeted pharmaceutical nanocarriers for cancer therapy and imaging. AAPS J 9: 128-147.

7. Danson S, Ferry D, Alakhov V, Margison J, Kerr D, et al. (2004) Phase I dose escalation and pharmacokinetic study of pluronic polymer-bound doxorubicin (SP1049C) in patients with advanced cancer. Br J Cancer 90: 2085-2091.

8. Matsumura Y, Hamaguchi T, Ura T, Muro K, Yamada Y, et al. (2004) Phase clinical trial and pharmacokinetic evaluation of NK911, a micelle-encapsulated doxorubicin. Br J Cancer 91:1775-1781.

9. Kim TY, Kim DW, Chung JY, Shin SG, Kim SC, et al. (2004) Phase I and pharmacokinetic study of Genexol-PM, a cremophor-free, polymeric micelleformulated paclitaxel, in patients with advanced malignancies. Clin Cancer Res 10: $3708-3716$

10. Sutton D, Nasongkla N, Blanco E, Gao J (2007) Functionalized micellar systems for cancer targeted drug delivery. Pharm Res 24: 1029-1046.

11. Lammers T, Hennink WE, Storm G (2008) Tumour-targeted nanomedicines: principles and practice. $\mathrm{Br} \mathrm{J}$ Cancer 99: 392-397.

12. Wang T, Petrenko VA, Torchilin VP (2010) Paclitaxel-loaded polymeric micelles modified with MCF-7 cell-specific phage protein: enhanced binding to target cancer cells and increased cytotoxicity. Mol Pharm 7: 1007-1014.

13. Reubi JC (2003) Peptide receptors as molecular targets for cancer diagnosis and therapy. Endocr Rev 24: 389-427.

14. Mori T (2004) Cancer-specific ligands identified from screening of peptidedisplay libraries. Curr Pharm Des 10: 2335-2343.

15. Thurber GM, Schmidt MM, Wittrup KD (2008) Factors determining antibody distribution in tumors. Trends Pharmacol Sci 29: 57-61.

16. Joshiand BP, Wang TD (2010) Exogenous Molecular Probes for Targeted Imaging in Cancer: Focus on Multi-modal Imaging. Cancers (Basel) 2: 1251 1287.

17. Petrenko V (2008) Evolution of phage display: from bioactive peptides to bioselective nanomaterials. Expert Opin Drug Deliv 5: 825-836.

18. Landon LA, Deutscher SL (2003) Combinatorial discovery of tumor targeting peptides using phage isplay. J Cell Biochem 90: 509-517.

19. Wang T, D'Souza GG, Bedi D, Fagbohun OA, Potturi LP, et al. (2010) Enhanced binding and killing of target tumor cells by drug-loaded liposomes modified with tumor-specific phage fusion coat protein. Nanomedicine (Lond) 5: 563-574.

20. Wang T, Kulkarni N, D'Souza GG, Petrenko VA, Torchilin VP (2011) On the mechanism of targeting of phage fusion protein-modified nanocarriers: only the binding peptide sequence matters. Mol Pharm 8: 1720-1728.

21. Jayanna PK, Bedi D, Gillespie JW, Delnnocentes P, Wang T, et al. (2010) Landscape phage fusion protein-mediated targeting of nanomedicines enhances their prostate tumor cell association and cytotoxic efficiency. Nanomedicine 6: 538-546.

22. Wang T, Yang S, Petrenko VA, Torchilin VP (2010) Cytoplasmic delivery of liposomes into MCF-7 breast cancer cells mediated by cell-specific phage fusion coat protein. Mol Pharm 7: 1149-1158.

23. Drummond DC, Meyer O, Hong K, Kirpotin DB, Papahadjopoulos D (1999) Optimizing liposomes for delivery of chemotherapeutic agents to solid tumors. Pharmacol Rev 51: 691-743. 
Citation: Wang T, Petrenko VA, Torchilin VP (2012) Optimization of Landscape Phage Fusion Protein-Modified Polymeric PEG-PE Micelles for Improved Breast Cancer Cell Targeting. J Nanomedic Nanotechnol S4:008. doi:10.4172/2157-7439.S4-008

Page 7 of 6

24. Noble CO, Kirpotin DB, Hayes ME, Mamot C, Hong K, et al. (2004) Development of ligand-targeted liposomes for cancer therapy. Expert Opin Ther Targets 8: 335-353.

25. Constantinides PP, Chaubal MV, Shorr R (2008) Advances in lipid nanodispersions for parenteral drug delivery and targeting. Adv Drug Deliv Rev 60: 757-767

26. Lukyanov AN, Torchilin VP (2004) Micelles from lipid derivatives of watersoluble polymers as delivery systems for poorly soluble drugs. Adv Drug Deliv Rev 56: $1273-1289$.

27. Wang T, Upponi R, Torchilin VP (2012) Design of multifunctional non-vira gene vectors to overcome physiological barriers: Dilemmas and strategies. Int J Pharm.

28. Hatakeyama $\mathrm{H}$, Akita $\mathrm{H}$, Harashima $\mathrm{H}$ (2011) A multifunctional envelope type nano device (MEND) for gene delivery to tumours based on the EPR effect: a strategy for overcoming the PEG dilemma. Adv Drug Deliv Rev 63: 152-160.

29. Weissig V, Whiteman KR, Torchilin VP (1998) Accumulation of protein-loaded long-circulating micelles and liposomes in subcutaneous Lewis lung carcinoma in mice. Pharm Res 15: 1552-1556

30. Torchilin VP (2004) Targeted polymeric micelles for delivery of poorly soluble drugs. Cell Mol Life Sci 61: 2549-2559.

31. Wang T, Kulkarni N, Bedi D, D'Souza GG, Papahadjopoulos-Sternberg B, et al. (2011) In vitro optimization of liposomal nanocarriers prepared from breast tumor cell specific phage fusion protein. J Drug Target 19: 597-605.

32. Krishnadas A, Rubinstein I, Onyuksel H (2003) Sterically stabilized phospholipid mixed micelles: in vitro evaluation as a novel carrier for water-insoluble drugs. Pharm Res 20: 297-302.

33. Onyuksel H, Ikezaki H, Patel M, Gao XP, Rubinstein I (1999) A novel formulation of VIP in sterically stabilized micelles amplifies vasodilation in vivo. Pharm Res 16: $155-160$.

34. Webster R, Didier E, Harris P, Siegel N, Stadler J, et al. (2007) PEGylated proteins: evaluation of their safety in the absence of definitive metabolism studies. Drug Metab Dispos 35: 9-16.
This article was originally published in a special issue, Nanotechnology: Targeted Drug Delivery handled by Editor(s). Dr. Sami M. Nazzal, University of Louisiana at Monroe, USA; Dr. Kytai Troung Nguyen, University of Texas at Arlington, USA 